\title{
SINGLE SPIN ASYMMETRIES IN ELECTROPRODUCTION AT CLAS
}

\author{
H. AVAKIAN AND L. ELOUADRHIRI* \\ Jefferson Lab, \\ 12000 Jefferson Ave., \\ Newport News, VA 23606, USA
}

\begin{abstract}
We present measurements of spin asymmetries in semi-inclusive processes in hard scattering kinematics using a $5.7 \mathrm{GeV}$ electron beam and the CEBAF Large Acceptance Spectrometer (CLAS) at JLab. Scattering of longitudinally polarized electrons off an unpolarized liquid-hydrogen and off a polarized $\mathrm{NH}_{3}$ targets was studied over a wide range of kinematics. Non-zero single-beam and single-target spin asymmetries have been observed in semi-inclusive pion production in hardscattering kinematics $\left(\mathrm{Q}^{2}>1.2 \mathrm{GeV}^{2}, \mathrm{~W}^{2}>4 \mathrm{GeV}^{2}\right)$. Systematic studies of factorization of $x$ and $z$ dependences have been done for different spin-dependent and spin-independent observables. No significant $x / z$ dependence has been observed within statistical uncertainties, which is consistent with factorization of hard scattering and fragmentation processes.
\end{abstract}

\section{Introduction}

Single-spin asymmetries (SSA) in azimuthal distributions of final state particles in semi-inclusive deep inelastic scattering (DIS) play a crucial role in the study of transverse momentum distributions of quarks in the nucleon and may provide access to the orbital angular momentum of quarks. Recently, significant SSAs were reported in semi-inclusive DIS (SIDIS) by the HERMES collaboration at HERA ${ }^{1,2}$ for longitudinally and transversely polarized targets, and by the CLAS collaboration at the Thomas Jefferson National Accelerator Facility (JLab) with a polarized beam ${ }^{3}$.

In recent years parton distribution functions have been generalized to contain information not only on the longitudinal but also on the transverse distributions of partons in a fast moving hadron. Eight independent transverse momentum dependent (TMD) parton distributions were identified ${ }^{4,5,6}$ at leading twist, with the transverse momentum $k_{T}$ of partons included,

${ }^{*}$ For the CLAS collaboration 
which are accessible in SIDIS. As shown recently in Ref. ${ }^{7}$, the interaction between the active parton in the hadron and the target spectators ${ }^{8,9,10}$ leads to gauge-invariant TMDs. Furthermore, the factorization for semi-inclusive scattering with hadrons in the current fragmentation region detected at low transverse momentum was verified to one-loop order in perturbative $\mathrm{QCD}^{11}$.

\section{Spin asymmetries in SIDIS with Polarized target and beam}

Assuming that the quark scattering and fragmentation processes factorize, the Bjorken- $x$ and $z$ (fraction of the energy of the virtual photon carried by the pion) dependences of the total cross section are given by the $x$ dependence of the distribution function and the $z$ dependence of the fragmentation function. Studies of dependences on $x$ and $z$ of pions in SIDIS thus provide a simple test of factorization.

No significant dependence on $x$ was observed for pion multiplicities as a function of $z$ within statistical uncertainties $(10-15 \%)$ already at beam energies as low as $4 \mathrm{GeV}^{3}$. A variety of possible observables to test the factorization is provided when measuring single and double polarized asymmetries as a function of $x$ or $z$ in different bins of $z$ and $x$ respectively.

A measurement of the double spin asymmetry $\left(A_{1 p}^{\pi+}\right)$ was performed at CLAS using an $\mathrm{NH}_{3}$ polarized target. The average beam polarization, frequently measured with a Møller polarimeter, was $0.73 \pm 0.03$ and the average target polarization was for $\mathrm{NH}_{3} 0.72 \pm 0.05$. The scattered electrons and pions were detected in CLAS ${ }^{12}$. No significant variation was observed for $A_{1 p}^{\pi+}$ as a function of $z$ within statistical uncertainties (see Fig. 1).

For a longitudinally polarized target the Collins fragmentation mechanism ${ }^{13}$ gives rise to a single-spin $\sin 2 \phi$ azimuthal asymmetry in leading order ${ }^{5,14}$. The measurements of average azimuthal moments are equivalent to measurements of corresponding spin asymmetries. Thus, the $\sin 2 \phi$ SSA of the cross section for longitudinally polarized target and unpolarized beam is defined as:

$$
\frac{1}{2} A_{U L}^{\sin 2 \phi}=<\sin 2 \phi>_{U L}=\frac{1}{P^{ \pm} N^{ \pm}} \sum_{i=1}^{N^{ \pm}} \sin 2 \phi_{i},
$$

where $P^{ \pm}$and $N^{ \pm}$are the polarization and number of events for \pm helicity state, respectively and $\phi$ is the azimuthal angle between the scattering plane 

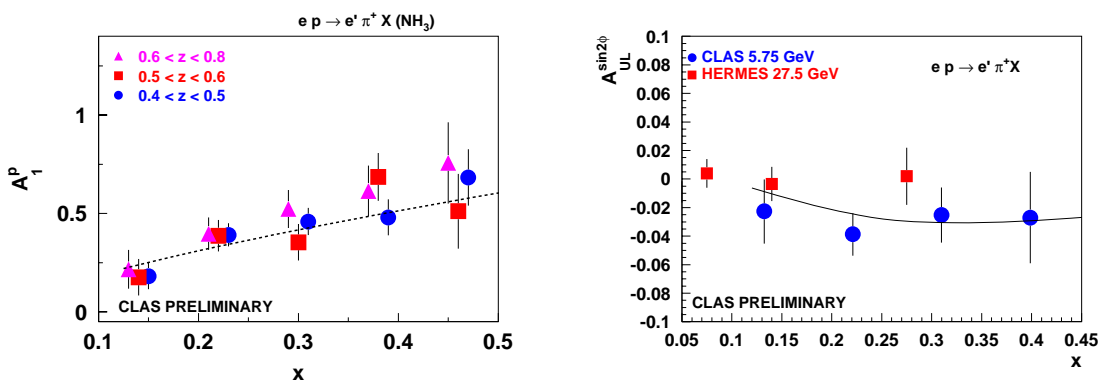

Figure 1. The double- (left panel) and single-spin (right panel) asymmetries as a function of $x$. The $\sin 2 \phi$ SSA is compared with the HERMES data ${ }^{1}$ and theory predictions $^{15}$.

formed by the initial and final momenta of the electron and the production plane formed by the transverse momentum of the observed hadron and the virtual photon. The final asymmetry is defined by the weighted average over two independent measurements for both helicity states.

A recent measurement by HERMES ${ }^{1}$ is consistent with a $\sin 2 \phi$ moment of zero. An experimentally observable effect has been predicted only at large $x$, a region well-covered by $\mathrm{JLab}^{15}$ (Fig. 1). The possible contribution from decaying $\rho$ s and detector acceptance are under study. The leading-twist distribution function $h_{1 L}^{\perp}(x)$, accessible in this measurement, describes the transverse polarization of quarks in a longitudinally polarized proton ${ }^{5,14}$.

Azimuthal moments of the SIDIS cross section with a longitudinally polarized target contain contributions both from the Sivers effect (T-odd distribution) and the Collins effect (T-odd fragmentation) ${ }^{13,8,9}$ and additional measurements are required to separate them. They include SSA measurements with transversely polarized target ${ }^{2}$ and measurements with polarized beam and unpolarized target.

The beam spin asymmetry is a higher twist effect and provides access to the elusive fifth structure function $H_{L T^{\prime}}$. It has three main contributions related to final state interactions (neglecting quark masses and higher order terms), involving convolutions of five unknown distribution and fragmentation functions. They were first identified by Levelt and Mulders ${ }^{16}$, Yuan $^{17}$ and Baccetta et $\mathrm{al}^{18}$.

A data set obtained with $5.7 \mathrm{GeV}$ electrons scattering off a 5-cm liquidhydrogen target (CLAS e16 data set) was used for beam SSA studies. It corresponds to an integral luminosity of $2.6 \times 10^{40} \mathrm{~cm}^{-2}$. The total number 
of events in the DIS range $\left(Q^{2}>1.2 \mathrm{GeV}^{2}, W^{2}>4 \mathrm{GeV}^{2}\right)$ selected by quality, vertex, acceptance, fiducial, and kinematic cuts was $\approx 7.8 \times 10^{6}$ for electron- $\pi^{+}$coincidences. The measured beam SSA $A_{L U}^{\sin \phi}$ at $5.7 \mathrm{GeV}$ is positive for a positive electron helicity in the range of $0.15<x<0.4$ (see Fig. 2). It is consistent with CLAS measurements at $4.3 \mathrm{GeV}^{3}$, and increases with $z$ (up to $z=0.8$ ) and with transverse momentum of the pion $P_{\perp}$, (Fig. 2).

Beam SSAs extracted at $4.3 \mathrm{GeV}^{3}$ after correction for a kinematic prefactor, which is different at CLAS and HERMES, are also consistent with preliminary HERMES data ${ }^{19}$ (right panel in Fig. 2), indicating that there is no significant dependence of beam SSA on beam energy. No significant $M_{X}$ $\left(e p \rightarrow e^{\prime} \pi^{+} X\right)$ dependence was observed, indicating that the asymmetry is not related to any specific remnant configuration (Fig. 2). This findings are consistent with predictions for factorization already at relatively low transverse momenta of hadrons ${ }^{20}$.

While the cut on the missing mass $\left(M_{X}>1.4 \mathrm{GeV}\right)$ removes most of the exclusive events from the semi-inclusive pion sample, it may still be diluted by exclusive vector meson production, which may contribute significantly in certain kinematic bins (Fig.3). The beam SSA extracted for $\pi^{+}$subsample from decaying $\rho^{0}$ s exhibits also a strong dependence on $z$ (see Fig.3). The pions from $\rho^{0}$ decays may be responsible for some part of the rise of the SSA at large $z(z>0.6)$.
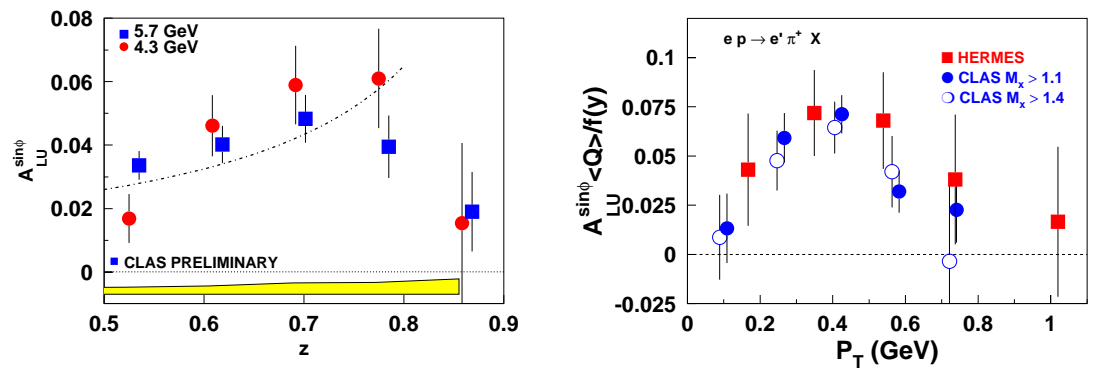

Figure 2. The beam-spin azimuthal asymmetry ( $\sin \phi$ moment of the cross section) extracted from hydrogen data at $5.7 \mathrm{GeV}$ (squares) and $4.3 \mathrm{GeV}$ (circles) as a function of $z$ in a range $0.1<x<0.4$ (left panel) and as a function of $P_{\perp}$ (right panel). The curve represents calculation performed assuming only the Sivers effect ${ }^{17}$ 

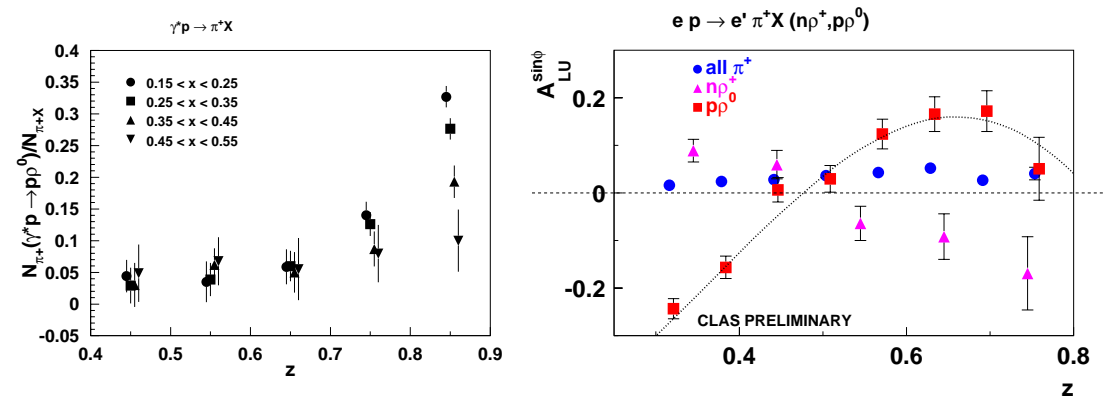

Figure 3. The fraction of $\pi^{+}$from exclusive $\rho$ decays in the single-pion sample (left panel) and their beam-spin azimuthal asymmetry $(\sin \phi$ moment of the cross section) extracted from hydrogen data at $5.7 \mathrm{GeV}$.

In conclusion, the data from CLAS experiments with $5.7 \mathrm{GeV}$ polarized electrons provide a unique possibility to examine the factorization hypothesis and study kinematic dependences of SSAs in semi-inclusive processes in hard scattering kinematics needed for separation of different contributions, and extraction of underlying, essentially unexplored, distribution functions.

\section{References}

1. HERMES collaboration (A. Airapetyan et al.), Phys. Rev. Lett. 84, 4047 (2000); Phys. Rev. D64, 097101 (2001).

2. HERMES collaboration (A. Airapetyan et al.), hep-ex/0408013.

3. CLAS Collaboration (H. Avakian et al.), hep-ex/0301005.

4. J. Ralston and D. Soper, Nucl. Phys. B152, 109 (1979)

5. P.J. Mulders and R.D. Tangerman, Nucl. Phys. B461, 197 (1996).

6. D. Boer and P. Mulders, Nucl. Phys. B 569, 505 (2000).

7. A. Belitsky, X. Ji and F. Yuan, Nucl. Phys. B 656, 165 (2003).

8. S. Brodsky et al., Phys. Lett. B 530, 99 (2002).

9. J. Collins, Phys. Lett. B 536, 43 (2002).

10. X. Ji, F. Yuan, Phys. Lett. B 543, 66 (2002).

11. X. Ji, J-P. Ma and F. Yuan, hep-ph/0404183.

12. B. Mecking et al., Nucl. Instrum. Meth. A503, 513 (2003).

13. J. Collins, Nucl. Phys. B396, 161 (1993).

14. A.M. Kotzinian and P.J. Mulders, Phys. Rev. D54 1229 (1996).

15. A. Efremov et al., Phys. Rev. D 67, 114014 (2003).

16. J. Levelt and P. J. Mulders, Phys. Lett. B338, 357 (1994).

17. F. Yuan, hep-ph/0310279.

18. A. Baccetta, P.J. Mulders and F. Pijlman, Phys.Lett. B595, 309, 2004

19. E. Avetisyan for HERMES Collaboration, hep-ph/0408002.

20. A. Idilbi, X. Ji and F. Yuan, hep-ph/0406302. 